\title{
Validation of Zero TE-MRA in the Characterization of Cerebrovascular Diseases: A Feasibility Study
}

\author{
(D) Shang, (D). Ye, (D)W. Dou, (D)X. Luo, (D). Qu, (D). Zhu, (D) H. Zhang, and (D). Wu
}

\begin{abstract}
BACKGROUND AND PURPOSE: Zero TE-MRA is less sensitive to field heterogeneity, complex flow, and acquisition noise. This study aimed to prospectively validate the feasibility of zero TE-MRA for cerebrovascular diseases assessment, compared with TOF-MRA.
\end{abstract}

MATERIALS AND METHODS: Seventy patients suspected of having cerebrovascular disorders were recruited. Sound levels were estimated for each MRA subjectively and objectively in different modes. MRA image quality was estimated by 2 neuroradiologists. The degree of stenosis (grades $0-4$ ) and the z-diameter of aneurysms (tiny group $\leq 3 \mathrm{~mm}$ and large group $>3 \mathrm{~mm}$ ) were measured for further quantitative analysis. CTA was used as the criterion standard.

RESULTS: Zero TE-MRA achieved significantly lower subjective perception and objective noise reduction (37.53\%). Zero TE-MRA images showed higher signal homogeneity ( $3.29 \pm 0.59$ versus $3.04 \pm 0.43$ ) and quality of venous signal suppression ( $3.67 \pm 0.47$ versus $2.75 \pm 0.46$ ). The intermodality agreement was higher for zero TE-MRA than for TOF-MRA (zero TE, 0.90; TOF, 0.81) in the grading of stenosis. Zero TE-MRA had a higher correlation than TOF-MRA (zero TE, 0.84; TOF, 0.74) in the tiny group and a higher consistency with CTA (intraclass correlation coefficient, 0.83 ; intercept, $-0.5084-1.1794$; slope -0.4952 to -0.2093 ) than TOF-MRA (intraclass correlation coefficient, 0.64 ; intercept, $0.7000-2.6133$; slope -1.0344 to -0.1923 ). Zero TE-MRA and TOF-MRA were comparable in the large group. Zero TE-MRA had more accurate details than TOF-MRA of AVM and Moyamoya lesions.

CONCLUSIONS: Compared with TOF-MRA, zero TE-MRA achieved more robust performance in depicting cerebrovascular diseases. Therefore, zero TE-MRA was shown to be a promising MRA technique for further routine application in the clinic in patients with cerebrovascular diseases.

ABBREVIATIONS: $\mathrm{ASL}=$ arterial spin-labeling; $\mathrm{AVM}=$ arteriovenous malformation; $\mathrm{MRA}=$ magnetic resonance angiography; $\mathrm{CTA}=$ computed tomography angiography; $C E$ = contrast-enhanced; $T O F=$ time-of-flight; $z T E=$ zero echo time; $M I P=$ maximum intensity projection; $V R=$ volume rendering; $M C A=$ middle cerebral artery; ICA = internal carotid artery

C erebrovascular diseases are the main causes of ischemic or hemorrhagic incidents within brain tissues and can lead to neurologic deficits or even death. Imaging of the cerebral vasculature plays a pivotal role in the initial diagnosis of cerebrovascular disorders, such as steno-occlusive arterial disease, cerebral an-

Received April 29, 2019; accepted after revision July 2.

From the Department of Radiology (S.S., J.Y., X.L., Q.Z., H.Z., J.W.), Clinical Medical College, Yangzhou University, Yangzhou, Jiangsu, China; and MR Research China (W.D., J.Q.), GE Healthcare, Beijing China.

This work was supported by a grant from the National Natural Science Foundation of China (NSFC 81571652), "333 Project” of Jiangsu Province (BRA2017154), Science and technology project of Yangzhou (YZ2018059).

Please address correspondence to Jingtao Wu, MD, Department of Radiology, Clinical Medical College, Yangzhou University, No. 98, Nantong West Road, Guangling, Yangzhou 225009, Jiangsu, China; e-mail: wujingtaodoctor@126.com

- Indicates open access to non-subscribers at www.ajnr.org

Indicates article with supplemental on-line photos.

http://dx.doi.org/10.3174/ajnr.A6173 eurysm, AVM, and Moyamoya disease, in addition to treatment decisions and follow-up evaluation. ${ }^{1,2}$ Although patients suspected of having cerebrovascular disease are best diagnosed with DSA or CTA due to their superior angiogram quality, ${ }^{3,4}$ these protocols place patients at risk of ionizing radiation and contrastinduced renal insufficiency.

MRA, a noninvasive and radiation-free imaging technique, has been proposed as a promising alternative for vascular disease assessment. ${ }^{5}$ In addition to conventional angiographic techniques, such as TOF-MRA and contrast-enhanced MRA, MRA is also able to use the principles of arterial spin-labeling (ASL) strategies. ${ }^{6}$ ASL-MRA was introduced as a noninvasive approach that uses inflowing blood as an endogenous contrast agent. Recent technical advances in ASL-MRA have dramatically expanded its clinical application. In particular, a newly developed zero TE radial ASL-MRA (zTE-MRA) method that integrates both a continuous ASL strategy and a zTE radial acquisition readout has dem- 


\begin{tabular}{|c|c|c|c|c|c|c|c|c|c|c|c|c|}
\hline & $\begin{array}{l}\text { TR/TE } \\
\text { (ms) }\end{array}$ & $\begin{array}{l}\text { Flip } \\
\text { Angle }\end{array}$ & $\begin{array}{l}\text { FOV } \\
(\mathrm{cm})\end{array}$ & Matrix & $\begin{array}{l}\text { Thickness } \\
\text { (mm) }\end{array}$ & Slices & NEX & $\begin{array}{l}\text { Bandwidth } \\
\text { (kHz) }\end{array}$ & Slabs & $\begin{array}{c}\text { Label } \\
\text { Duration } \\
\text { (sec) }\end{array}$ & Coverage & $\begin{array}{c}\text { Time } \\
\text { (min:sec) }\end{array}$ \\
\hline zTE-MRA & $862 / 0.016$ & $3^{\circ}$ & $15 \times 15$ & $166 \times 166$ & 1.2 & 320 & 1 & 31.25 & - & 2 & Calvarium-mandible & $5: 48$ \\
\hline TOF-MRA & $25 / 3.4$ & $15^{\circ}$ & $30 \times 24$ & $320 \times 256$ & 1.4 & 256 & 1 & 41.67 & 3 & - & $\begin{array}{l}\text { Cingulate cortex- } \\
\text { mesencephalon }\end{array}$ & 5:08 \\
\hline
\end{tabular}

Note:- - indicates no data available; TR, repetition time; TE, echo time; FOV, field of view; NEX, number of excitation.

Table 2: Parameters of CTA sequences

\begin{tabular}{|c|c|c|c|c|c|c|c|c|c|c|}
\hline & Collimation & Pitch & $\begin{array}{l}\text { FOV } \\
(\mathrm{cm})\end{array}$ & $\begin{array}{c}\text { Gantry } \\
\text { Rotation } \\
\text { Time (ms) }\end{array}$ & $\begin{array}{l}\text { Thickness } \\
\text { (mm) }\end{array}$ & Slices & $\begin{array}{c}\text { Tube Voltage } \\
\text { (kV[peak]) }\end{array}$ & $\begin{array}{l}\text { Tube } \\
\text { Current } \\
\text { (mAs) }\end{array}$ & $\begin{array}{l}\text { Dose-Length } \\
\text { Product } \\
(\mathrm{mGy} \times \mathrm{cm})\end{array}$ & Coverage \\
\hline CTA & $128 \times 0.625$ & 0.758 & $17 \times 17$ & 400 & 0.6 & 715 & $100-120$ & $100-450$ & $220-608$ & Aortic arch-vertex \\
\hline
\end{tabular}

onstrated potential for the follow-up assessment of coiled aneurysms, ${ }^{7-10}$ highlighting the clinical value of this approach.

However, it remains unclear whether this technique is also useful for the assessment of other cerebrovascular disorders. Meanwhile, the acoustic noise reduction of zTE-MRA has still not been assessed systematically, though this approach was described as silent. ${ }^{7-9}$ To explore this issue, we applied this novel, nonenhanced MRA approach in a clinical population with suspected cerebrovascular diseases. Sound-level assessment and imaging properties were systematically assessed and compared with those obtained using TOF-MRA. In addition, CTA measurements were also performed and used as the criterion standard.

\section{MATERIALS AND METHODS \\ Patient Cohort}

A single-center prospective study was performed with the approval from local institutional review board of Clinical Medical College, Yangzhou University (2017-KY081). Patients suspected of having cerebrovascular disorders were enrolled in this study from May 2017 to February 2018. Cerebrovascular disorders were suspected for the first time by 2 experienced neurologists (Yong Zhen, with 24 years of experience; Long Yu, with 30 years of experience) on the basis of clinical symptoms (eg, dizziness, headache, paralysis, paresthesia, conscious disturbance, dysmnesia, dementia, and other psychiatric symptoms) and routine head CT examinations (eg, infarction, hemorrhage, encephalatrophy, and other lesions of abnormal density). Written informed consent was obtained from each participant before MR imaging.

The following criteria for patient exclusion were applied in this study: 1) contraindication for MR imaging, including severe claustrophobia, ferromagnetic foreign bodies, or electronic implants; 2) other intracranial lesions, such as tumor or hydrocephalus (traumatic or tumor-related); 3 ) an acute or subacute window for a vascular event (relatively large acute cerebral infarction or acute cerebral infarction of the brain stem on DWI); 4) additional conditions that could potentially cause severe movement or being uncooperative, such as psychiatric disorders or congenital/ acquired deafness; 5) estimated glomerular filtration rate $<60$ $\mathrm{mL} / \mathrm{min} / 1.73 \mathrm{~m}^{2}$; and 6 ) younger than 18 years of age. Finally, 69 patients ( 38 men and 31 women; range, $20-83$ years of age; mean age, $60.04 \pm 13.70$ years) were enrolled in this study. One patient had subarachnoid hemorrhage caused by aneurysm and underwent only MRA because of an allergy to iodinated contrast media. The MRA images (On-line Fig 1) were thus used only for image- quality measurement. Finally, both MRA and CTA were successfully performed in 68 patients.

\section{Image Acquisition}

MR imaging measurements (DWI, MRA) were obtained 24 hours before CTA acquisition on a 3T MR imaging scanner (Discovery MR750w; GE Healthcare, Milwaukee, Wisconsin) using a 24channel head-neck united coil. The corresponding scan parameters and source images are shown in Table 1 and On-line Fig 2.

CTA was acquired on a 64-slice spiral CT scanner (LightSpeed VCT; GE Healthcare) using a contrast medium (iodixanol, Visipaque, $320 \mathrm{mg} \mathrm{I/mL;} \mathrm{GE} \mathrm{Healthcare,} \mathrm{Piscataway,} \mathrm{New} \mathrm{Jersey).}$ The scanning protocol was described in a previously reported study. ${ }^{11}$ The corresponding scan parameters are shown in Table 2.

\section{Sound Assessment of MRA Measurements}

MRAs were performed in random order and separated by a 5-minute interval during scanning.

After each measurement, the patient was asked to rate the sound experience subjectively on a 5 -point scale ${ }^{12}: 0=$ no noise, $1=$ mild noise, $2=$ moderate noise, $3=$ loud noise, $4=$ very loud noise, and $5=$ unacceptable noise/cancellation of MR imaging.

Objective measurements were obtained in an ambient mode (without scanning) and scanning mode using a sound-level meter (Type AS804; Smart Sensor, Guangdong, China) that was placed $1 \mathrm{~m}$ from the gantry. Each measurement period lasted for $30 \mathrm{sec}-$ onds, and measurements were repeated 5 times in different directions.

\section{Image Analysis}

The maximum intensity projection (MIP) and volume-rendering (VR) methods were used to reconstruct both MRA and CTA data by a neuroradiologist (Q.Z., with 9 years of experience) using a commercially available dedicated workstation (Advantage Workstation, software Version 4.6; GE Healthcare) We made the following standard projections of MIP and VR: 1) coronal view, 2) lateral view, and 3 ) the optimal projection used at the lesions.

The corresponding CTA images (source image, MIP, and VR) were independently and blindly evaluated by 2 experienced neuroradiologists (observer C, J.W., with 27 years of experience; observer D, X.L., with 16 years of experience) at separate time points. The corresponding MRA images (source image, MIP, and VR) were independently and blindly evaluated by 2 experienced neuroradiologists (observer A, H.Z., with 25 years of experience; ob- 
server B, J.Y., with 20 years of experience)) at separate time points. All images were scored to determine signal homogeneity, lesion conspicuity, quality of venous signal suppression, and diagnostic confidence. A previously reported 4-point scale was applied ${ }^{13,14}$ : $4=$ excellent (excellent-quality diagnostic information with a clearly detailed vascular architecture, no artifacts), $3=$ good (good-quality diagnostic information with adequate delineation of the vascular architecture, minimal artifacts), 2 = poor (poorquality diagnostic information with ordinary delineation of the vascular architecture, moderate artifacts), and $1=$ not visible (almost no signal of the vascular architecture, severe artifacts). The time interval between the qualitative analyses was 4 weeks. In cases of disagreement, a consensus was established between observers.

Observers were also asked to record additional vascular disease findings based on MRA and CTA images. The measurements were performed on the VR images. For further quantitative analysis, the degree of stenosis was graded according to the standard from NASCET: 0 for $<9 \%$, one for $<30 \%$, two for 30\%-69\%, three for $70 \%-99 \%$, and 4 for $100 \%$. In addition, aneurysms were divided into tiny $(\leq 3 \mathrm{~mm})$ and large $(>3 \mathrm{~mm})$ groups according to the long-axis diameters of the lesions as determined on CTA images.

\section{Statistical Analysis}

All statistical analyses were performed using SPSS 19.0 software (IBM, Armonk, New York).

The differences in subjective noise-level and image-quality ratings between MRAs were analyzed using Wilcoxon signed rank tests. One-way analysis of variance tests and least significant dif-

Table 3: Intensity of sound levels in different modes for zTE-MRA and TOF-MRA

\begin{tabular}{cccc}
\hline Mode & Sound Level (dB) & $\boldsymbol{F}$ & $\boldsymbol{P}$ \\
\hline Ambient & $54.89 \pm 0.41^{\mathrm{a}}$ & $11,824.06$ & $<.001$ \\
zTE-MRA & $58.01 \pm 0.32^{\mathrm{ab}}$ & & \\
TOF-MRA & $92.86 \pm 0.64^{\mathrm{b}}$ & & \\
\hline
\end{tabular}

${ }^{a}$ A significant difference compared with the TOF-MRA mode.

${ }^{\mathrm{b}}$ A significant difference compared with the ambient mode.

Table 4: Cross-table of stenosis grade from observers for zTE-MRA $(n=44)^{a}$

\begin{tabular}{lcccccc}
\hline \multirow{2}{*}{ zTE-MRA } & \multicolumn{7}{c}{ zTE-MRA B Grade } \\
\cline { 2 - 7 } A Grade & $\mathbf{0}$ & $\mathbf{1}$ & $\mathbf{2}$ & $\mathbf{3}$ & $\mathbf{4}$ & Total \\
\hline 0 & 1 & 0 & 0 & 0 & 0 & $1(2.27 \%)$ \\
1 & 0 & 7 & 3 & 0 & 0 & $10(22.73 \%)$ \\
2 & 0 & 0 & 14 & 0 & 0 & $14(31.82 \%)$ \\
3 & 0 & 0 & 4 & 8 & 0 & $12(27.27 \%)$ \\
4 & 0 & 0 & 0 & 1 & 6 & $7(15.91 \%)$ \\
Total & $1(2.27 \%)$ & $7(15.91 \%)$ & $21(47.73 \%)$ & $9(20.45 \%)$ & $6(13.64 \%)$ & 44 \\
\hline
\end{tabular}

${ }^{a}$ Grading criterion: NASCET. Data represent the number of cases. A and B are observers A and B.

Table 5: Cross-table of stenosis grade from observers for TOF-MRA $(n=44)^{a}$

\begin{tabular}{|c|c|c|c|c|c|c|}
\hline \multirow{2}{*}{$\begin{array}{c}\text { TOF-MRA } \\
\text { A Grade }\end{array}$} & \multicolumn{6}{|c|}{ TOF-MRA B Grade } \\
\hline & 0 & 1 & 2 & 3 & 4 & Total \\
\hline 0 & 0 & 0 & 0 & 0 & 0 & 0 (0.00\%) \\
\hline 1 & 1 & 9 & 0 & 0 & 0 & $10(22.73 \%)$ \\
\hline 2 & 0 & 1 & 8 & 4 & 0 & $13(29.55 \%)$ \\
\hline 3 & 0 & 0 & 4 & 7 & 0 & $11(25.00 \%)$ \\
\hline 4 & 0 & 0 & 0 & 2 & 8 & $10(22.73 \%)$ \\
\hline Total & $1(2.27 \%)$ & 10 (22.73\%) & 12 (27.27\%) & $13(29.55 \%)$ & 8 (18.18\%) & 44 \\
\hline
\end{tabular}

${ }^{a}$ Grading criterion: NASCET. Data represent the number of cases. A and B are observers A and B. ference tests were used to estimate the difference in the objective sound-level measurements between MRAs.

For the stenosis analysis, the Spearman correlation coefficient $(r)$ was used to evaluate the correlation of each MRA to CTA. Weighted $\kappa$ statistics were used to assess interobserver agreement and intermodality agreement between the results obtained with each MRA and CTA.

The aneurysm measurements obtained on MRA were correlated with those obtained on CTA using a Pearson analysis. BlandAltman analysis and intraclass correlation coefficients were used to test the consistency of measurements obtained using the MRAs and CTAs. A weighted $\kappa$ value or an intraclass correlation coefficient higher than 0.81 was interpreted as excellent agreement, while $0.61-0.81$ indicated good, $0.41-0.60$ indicated moderate, $0.21-0.40$ indicated fair, and $<0.21$ indicated poor agreement. The $95 \%$ confidence interval was calculated. A $P$ value $<.05$ was

\section{RESULTS}

\section{Patient Cohort}

CTA revealed 32 steno-occlusive cases in 26 patients, 31 cerebral aneurysm cases in 22 patients, 11 steno-occlusive cases and 8 cerebral aneurysm cases in 8 patients, 2 patients with AVMS, 3 patients with Moyamoya disease, and 7 patients without evident vascular diseases. The distribution of the steno-occlusive arteries (cases) was as follows: middle cerebral artery (MCA), 18; internal carotid artery (ICA), 10; posterior cerebral artery, 6; anterior cerebral artery, 5; vertebral artery, 2; and basilar artery 2 . The distribution of the aneurysms was as follows: posterior communicating artery, 14; anterior communicating artery, 10; ICA, 7; MCA, 4; anterior cerebral artery, 2; posterior inferior cerebellar artery, 1; and vertebral artery, 1 . The $2 \mathrm{AVM}$ lesions were located in the left MCA and right ICA (1 each). The 3 Moyamoya lesions were located at the origin of a unilateral MCA.

\section{Sound-Level Assessment}

Mean sound-level perception based on subjective sound experience was significantly lower on zTE-MRA than on TOF-MRA $(1.36 \pm 0.48$ versus $4.17 \pm 0.75, P=$ $.001)$. The intensities of sound measured in different modes are shown in Table 3. Noise levels recorded during zTE-MRA scanning were $34.85 \mathrm{~dB}$ (37.53\%) lower in sound intensity. In addition, there was a slight increase $(3.12 \mathrm{~dB}, 5.68 \%$, $P<.001)$ in sound levels over the ambient mode.

\section{Image-Quality Evaluation of zTE- and TOF-MRA}

The mean scores for signal homogeneity $(3.29 \pm 0.59$ versus $3.04 \pm 0.43, P=.03)$ and the quality of venous signal suppression $(3.67 \pm 0.47$ versus $2.75 \pm 0.46$, $P=.01)$ were significantly higher for zTE-MRA than for TOF-MRA. zTEMRA was comparable with TOF-MRA in lesion conspicuity $(3.21 \pm 0.51$ versus 
$3.06 \pm 0.45, P=.08)$ and diagnostic confidence $(3.23 \pm 0.46$ versus $3.05 \pm 0.44, P=.06$ ). Additionally, the peripheral vessels presented on zTE-MRA were less well-defined than on TOF-MRA.

\section{Quantitative Analysis of zTE- and TOF-MRA Images}

The interobserver agreement for CTA was excellent (weighted $\kappa$, 0.94; 95\% CI, 0.90-0.99). CTA revealed eleven (25\%) cases with grade 1 , thirteen $(29.55 \%)$ with grade 2 , twelve $(22.27 \%)$ with grade 3 , and seven $(15.91 \%)$ with grade 4 . One case $(2.27 \%)$ was wrongly categorized as grade 1 by TOF-MRA, while zTE-MRA, consistent with CTA, showed that there was no steno-occlusive lesion (On-line Fig 3).

The correlations of the results obtained using each MRA with CTA results were both high (zTE, $r=0.94$; TOF, $r=0.92$ ). There was excellent interobserver agreement for zTE-MRA (weighted $\kappa$ : 0.92; 95\% CI, 0.87-0.98) and TOF-MRA (weighted $\kappa: 0.89 ; 95 \%$ CI, $0.84-0.96)$. The intermodality agreement between zTE-MRA and CTA was excellent (weighted $\kappa: 0.90 ; 95 \%$ CI, 0.82-0.99), and it was good (weighted $\kappa: 0.81 ; 95 \%$ CI, 0.71-0.92) between TOFMRA and CTA. These data are shown in Tables 4-6. Some lesions, especially at a critical point between 2 grades, may have been overestimated on TOF-MRA, while on ZTE-MRA, the stenosis was equal to that observed on CTA (Fig 1).

All aneurysms (39/39, 100\%) were detected by zTE-MRA. On

Table 6: Classification of stenosis grade from observers for zTE-MRA, TOF-MRA, and CTA $(n=44)^{\mathrm{a}}$

\begin{tabular}{lccc}
\hline Stenosis Grade & zTE-MRA & TOF-MRA & CTA \\
\hline 0 & $1(2.27 \%)$ & $0(0.00 \%)$ & $1(2.27 \%)$ \\
1 & $11(25.00 \%)$ & $10(22.73 \%)$ & $11(25.00 \%)$ \\
2 & $12(27.27 \%)$ & $12(27.27 \%)$ & $13(29.55 \%)$ \\
3 & $11(25.00 \%)$ & $11(25.00 \%)$ & $10(22.73 \%)$ \\
4 & $9(20.45 \%)$ & $11(25.00 \%)$ & $9(20.45 \%)$ \\
\hline
\end{tabular}

${ }^{a}$ Grading criterion: NASCET. Data represent the number of cases.
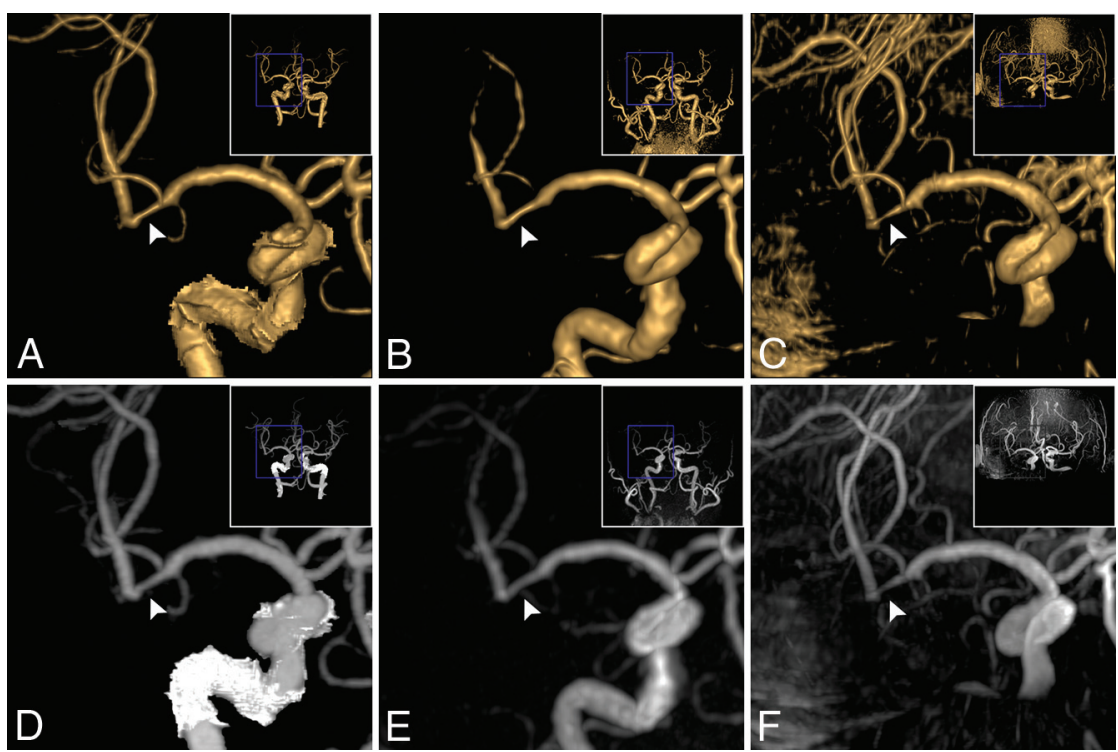

FIG 1. Coronal projection of stenosis in the right MCA M2 segment (a 74-year-old man). A stenosis $(34 \%$, grade 2$)$ was observed on VR of CTA ( $A$, white arrowhead) and on VR of zTE-MRA ( $B$, white arrowhead); the stenosis (32\%, grade 2) was equal to that on CTA. The stenosis on VR of TOF (C, white arrowhead) was overestimated (72\%, grade 3$)$. In correspondence with MIP of CTA ( $D$, white arrowhead), flow signal in the stenosis lesion was homogeneous on MIP of zTE-MRA (E, white arrowhead, score 4$)$ and was heterogeneous on TOF-MRA ( $F$, white arrowhead, score 3$)$.
TOF-MRA, 1 case $(2.56 \%)$ was missed due to limited coverage. Thus, there were 23 cases $(23 / 38,60.53 \%)$ in the large group and 15 cases $(15 / 38,39.47 \%)$ in the tiny group. The data are shown in Table 7. In the tiny group, according to Bland-Altman analysis, zTE MRA was equal to CTA, whereas TOF-MRA was not. In the large group, according to Bland-Altman analysis, zTE-MRA and TOF-MRA were equal to CTA. In the tiny group, zTE-MRA was more sensitive than TOF-MRA for the depiction of tiny aneurysms (Fig 2). In the large group, zTE-MRA was similar to TOFMRA in the depiction of large aneurysms (On-line Fig 4).

Because of their distinct characteristics, the 2 patients with $\operatorname{AVMs}(2 / 2,100 \%)$ and the 3 with Moyamoya disease (3/3, 100\%) were all diagnosed by observers using both MRA sequences. However, zTE-MRA was superior to TOF-MRA with regard to imaging details, such as the depiction of the nidus, draining vein, and venous sinus in AVMs (Fig 3) and stenosed arteries and developed collateral vessels in Moyamoya disease (Fig 4).

\section{DISCUSSION}

In this study, by comparing zTE-MRA with TOF-MRA, we demonstrate that zTE-MRA was able to depict cerebrovascular diseases (steno-occlusive arterial disease, cerebral aneurysm, AVM, and Moyamoya disease) with dramatically reduced acoustic noise, higher signal homogeneity, and higher quality of venous signal suppression. Interobserver and intermodality agreement was higher for zTE-MRA than for TOF-MRA for stenosis grading, indicating that zTE-MRA is a robust method for stenosis assessment. In addition, zTE-MRA provided more accuracy with regard to aneurysm-diameter measurement and morphologic assessment, especially in smaller aneurysms. zTE-MRA was superior to TOF-MRA in the identification of lesion details in both AVM and Moyamoya disease, despite the relatively small sample size.

Currently, TOF-MRA is the MR imaging technique conventionally used to depict intracranial arteries. However, there are known major limitations to the use of TOF-MRA for the delineation of cerebrovascular disorders, such as pseudoocclusions, ${ }^{15}$ the inaccurate detection of aneurysms ${ }^{16}$ and the nidus and draining veins in AVMs, ${ }^{17}$ and collateral vessels in Moyamoya disease. ${ }^{18}$ The resultant signal losses are mainly due to saturation secondary to slow flow or phase-dispersion effects caused by turbulent flow, ${ }^{19}$ which account for the overestimation or inaccuracy of TOF-MRA observed in our study. In contrast to TOF-MRA, zTE-MRA showed some encouraging characteristics as a nonenhanced MRA approach. Continuous ASL-based angiography was introduced as an endogenous contrast tracer in zTE-MRA to magnetically label inflowing blood, and this placed no constraint on the direction of blood flow. ${ }^{6}$ Thus, zTE-MRA has the potential to visualize slow flow and is independent of flow direction, features especially important for evaluating the 
Table 7: Statistical analyses of aneurysms measured on MRA for groups

\begin{tabular}{ccccccc}
\hline Group & MRA & Sum & $\boldsymbol{r}^{\mathbf{a}}$ & ${\text { Intercept }(95 \% \mathrm{Cl})^{\mathbf{b}}}$ & Slope $(95 \% \mathrm{Cl})^{\mathbf{b}}$ & ICC \\
\hline Group tiny $^{\mathrm{c}}$ & zTE & 15 & 0.84 & $-0.5084-1.1794$ & $-0.4952-0.2093$ & $0.83 ; 95 \% \mathrm{Cl}, 0.57-0.94$ \\
& TOF & 15 & 0.74 & $0.7000-2.6133$ & -1.0344 to -0.1923 & $0.64 ; 95 \% \mathrm{Cl}, 0.21-0.86$ \\
Group large $^{\mathrm{d}}$ & zTE & 23 & 0.98 & $-0.6059-0.2130$ & $-0.02625-0.1200$ & $0.98,95 \% \mathrm{Cl}, 0.97-0.99$ \\
& TOF & 23 & 0.95 & $-1.1571-0.3062$ & $-0.01380-0.2520$ & $0.95,95 \% \mathrm{Cl}, 0.89-0.98$ \\
\hline
\end{tabular}

Note:-ICC indicates intraclass correlation coefficient.

a Pearson correlation coefficient.

${ }^{\mathrm{b}}$ Intercept and slope are by Bland-Altman analysis.

${ }^{\circ}$ Group tiny $\leq 3 \mathrm{~mm}$.

${ }^{\mathrm{d}}$ Group large $>3 \mathrm{~mm}$.
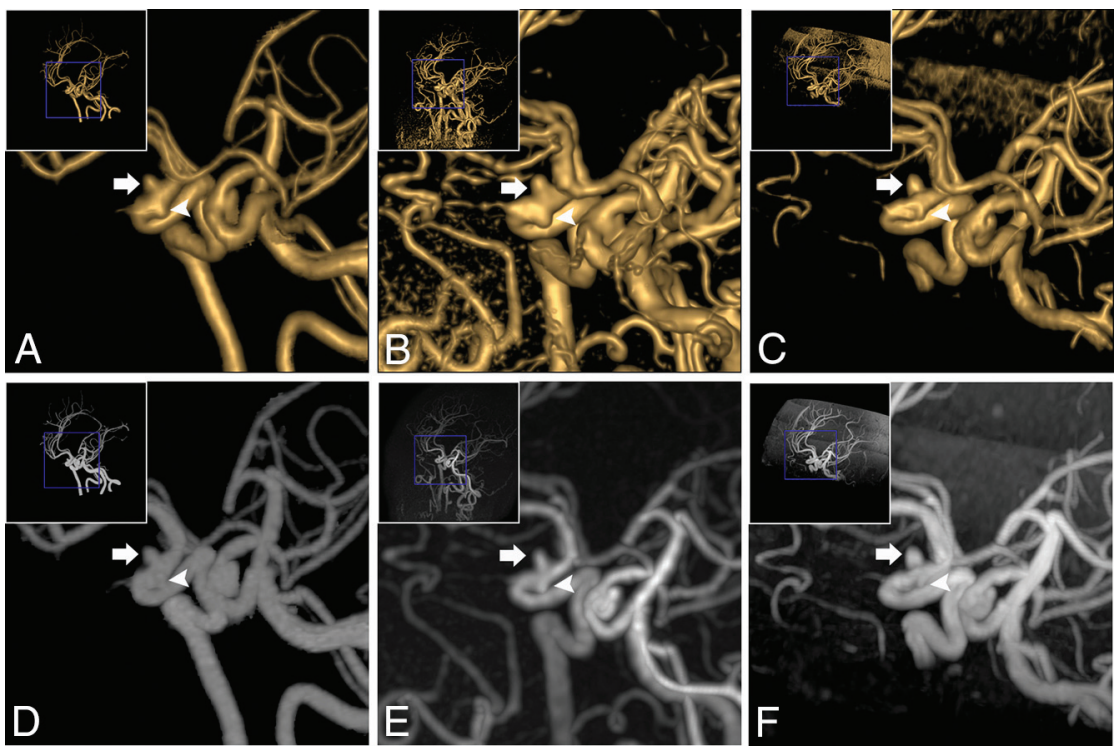

FIG 2. Oblique projection of aneurysms in the right ICA siphon segment (a 63-year-old woman). Two aneurysms were found on VR of CTA $(A)$ : The diameters were $3.0 \times 3.2 \mathrm{~mm}$ (white arrow) and $2.4 \times 2.1 \mathrm{~mm}$ (white arrowhead), respectively. Equal findings were observed on VR of zTE $(B, 3.3 \times$ $3.5 \mathrm{~mm}$, white arrow; $2 . \times 2.4 \mathrm{~mm}$, white arrowhead). On VR of TOF-MRA (C), the large one $(3.1 \times$ $3.4 \mathrm{~mm}$, white arrow) was equal to the one on CTA. However, the tiny one was not evident $(1.5 \times 1.4 \mathrm{~mm}$, white arrowhead). Concerning MIP of CTA (D, white arrow and white arrowhead), the same results were observed on MIP of ZTE-MRA (E, white arrow and white arrowhead) and TOF-MRA ( $F$, white arrow and white arrowhead).

flow in severe stenosis (grade 3), microaneurysms, and collateral vessels.

Acoustic noise induced by rapid switching of gradient coils during long scan times is still the main drawback that causes patient discomfort and movement. ${ }^{20-22}$ The zTE technique, which uses a sequence solution, attenuates acoustic noise by reducing the slew rate and enabling minimal gradient variations. ${ }^{23,24} \mathrm{Al}-$ ibek et $\mathrm{al}^{12}$ assessed the noise level and image quality of silent T1-weighted sequences on a clinical 3T MR imaging scanner. However, whether a similar amount of acoustic noise reduction could be achieved using zTE-MRA remains unclear because an extra ASL-based angiography was integrated into this approach. In this study, patients were much more comfortable during zTEMRA than TOF-MRA, and the reduction in sound intensity between the 2 was comparable with that of a silent T1-weighted sequence. Thus, zTE-MRA would be an acceptable alternative angiography method for these patients.

In addition to acoustic noise attenuation, a zTE inside-out radial acquisition could also minimize the phase dispersion of the labeled blood flow signal in the voxel space, thereby making zTEMRA insensitive to turbulent flow. ${ }^{9,25}$ The flow signal inside the large aneurysms was more homogeneous on zTE-MRA than on TOF-MRA in our study, though the correlations with CTA were both high and the consistency with CTA was excellent for the large group.

Susceptibility artifacts, which occur mostly in regions adjacent to the skull base or cavernous sinus, always induce signal loss as a result of air-soft tissue or bone-soft tissue boundary interference. ${ }^{26}$ Because of its use of a constant gradient and a long readout duration, zTE-MRA is less susceptible to field inhomogeneity and eddy current and can acquire more homogeneous flow signals in vascular lesions, such as stenosis or aneurysms in the ICA siphon segment. Moreover, zTE-MRA is less sensitive than TOF-MRA to motion effects, ${ }^{27}$ and it therefore minimizes the misregistration and degradation of image quality and achieves improved diagnostic performance.

According to the principles of the ASL strategy used by zTE-MRA, images are acquired via subtraction to yield an angiographic image with nearly zero background. ${ }^{7}$ The efficiency of postprocessing (ie, VR or MIP reconstructions) can be improved with automatic subtraction, whereas the artifacts caused by skull and brain tissue on TOF-MRA must be manually removed. Furthermore, zTE-MRA demonstrated effective background suppression while a search for aneurysms was performed in the setting of subarachnoid hemorrhage in the presence of residual T1-bright blood. An optimal postlabel delay was set, ${ }^{6,28}$ ensuring that the labeled bolus flowed into the target tissue in the imaging region and that the labeled protons lost their signal before entering the veins. Thus, better diagnostic image quality was achieved due to an acceptable signalto-noise ratio and the elimination of vein contamination. Additionally, zTE-MRA can detect arteriovenous shunting on the basis of this technical feature. In this study, we identified a huge AVM and found that zTE-MRA was capable of revealing its tubular structures, nidus, and draining veins and even a venous sinus.

Contrast-enhanced MRA is another commonly used noninvasive MRA approach performed by administrating a T1-shortening paramagnetic contrast medium. Despite the controversy related to its diagnostic accuracy, ${ }^{15,16}$ contrast-enhanced MRA has 

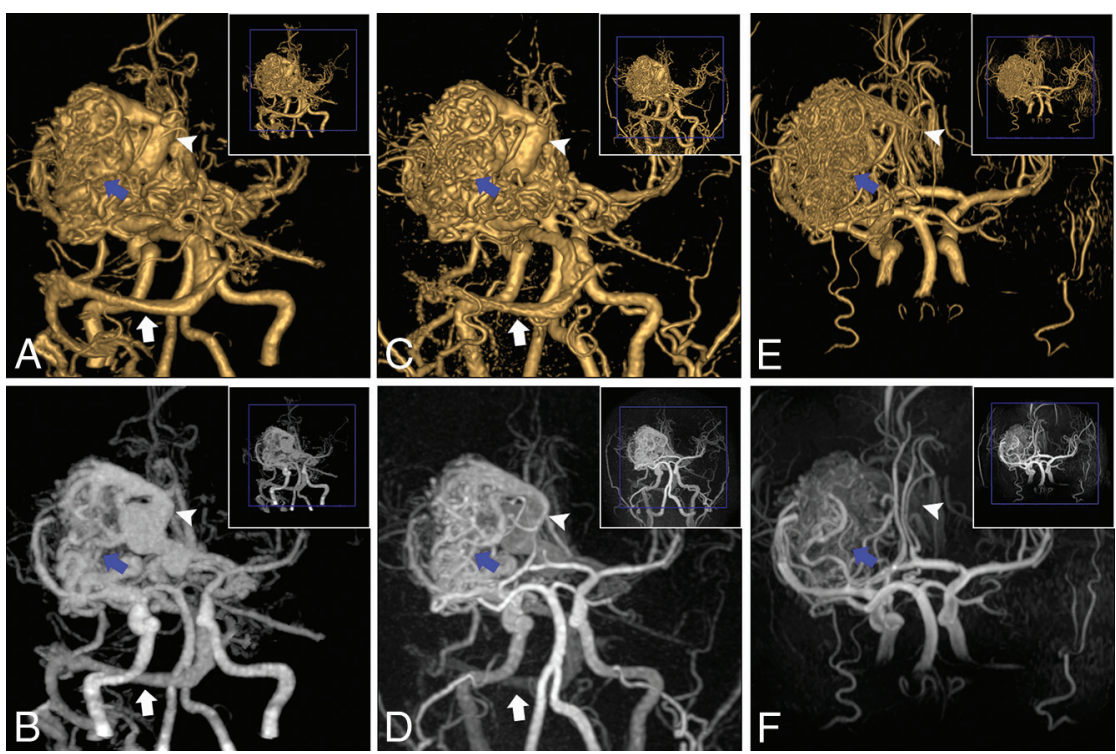

FIG 3. An AVM in the left MCA Ml segment (a 27-year-old man). For the AVM, the nidus (blue arrow), draining vein (white arrowhead), and venous sinus (transverse sinus and sigmoid sinus, white arrow) were clearly depicted on VR and MIP of CTA ( $A$ and $B$ ) and ZTE-MRA ( $C$ and D), whereas they were not well-defined (nidus, blue arrow; draining vein, white arrowhead) and were missed (venous sinus) on VR and MIP of TOF-MRA ( $E$ and $F$ ).
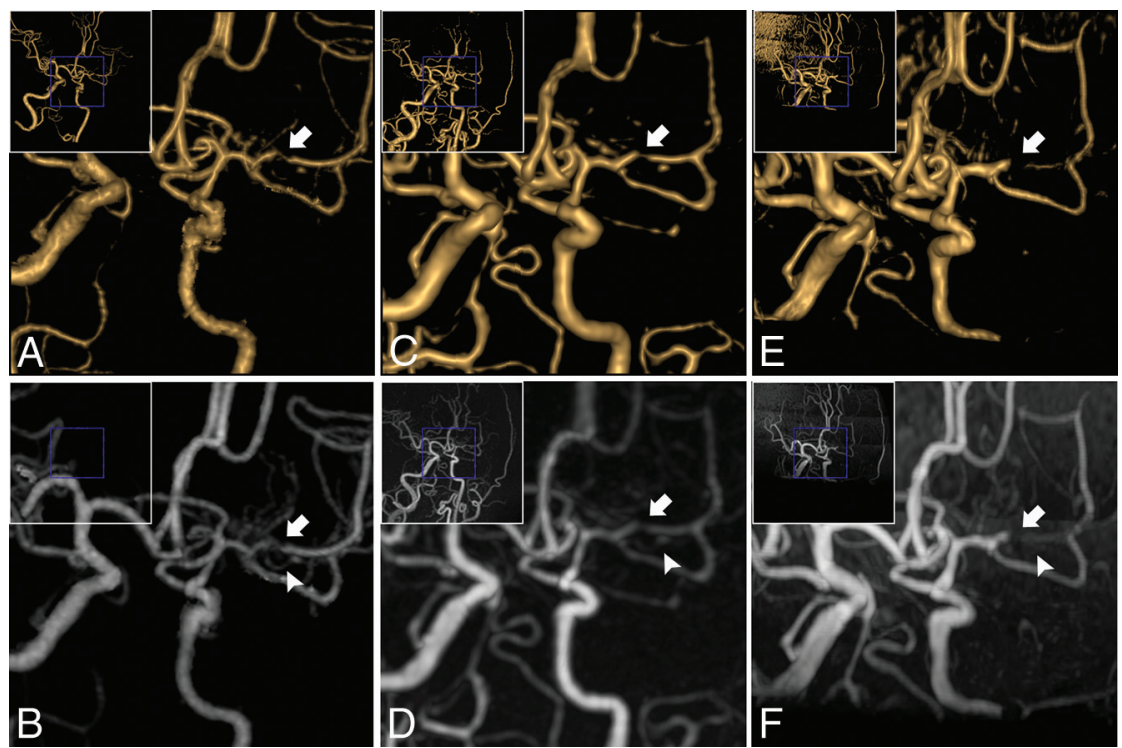

FIG 4. Oblique projection of Moyamoya disease in the left MCA M1 segment (a 43-year-old woman). The stenosed MCA (white arrowhead) and developed collateral vessels (white arrow) were scanned on VR and MIP of CTA ( $A$ and $B$ ) and zTE-MRA ( $C$ and $D)$. A false occlusion in MCA (white arrowhead) and ill-defined collateral vessels (white arrow) were seen on VR and MIP of TOF-MRA ( $E$ and $F$ ).

demonstrated some superiorities over TOF-MRA, such as it is less vulnerable to blood flow disturbances, has a reduced risk of movement artifacts, and provides large coverage for additional findings, while TOF-MRA also has some advantages over contrastenhanced MRA in that it requires no exogenous contrast agent and is repeatable and easy to manipulate. Although our results indicate that zTE-MRA integrates these potential aspects beyond the reduction of venous artifacts, these characteristics need to be verified by further research.

In the present study, we demonstrated that zTE-MRA is a robust sequence that maintains both zTE-like and ASL-like charac- teristics. Several studies ${ }^{7-10}$ have focused on zTE-MRA in the follow-up imaging of various aneurysm embolizations and have revealed that this method achieves higher image quality than is obtained by TOF-MRA. Our study enriches our knowledge of zTE-MRA and highlights the clinical usefulness in evaluating cerebrovascular diseases.

Holdsworth et $\mathrm{al}^{24}$ noted that zTEMRA is more prone to blurring of the vessel edge because of center-out radial sampling, as shown in Fig 1 and our previous study. ${ }^{10} \mathrm{Qu}$ et al ${ }^{29}$ implemented a hybrid ASL strategy in zTE-MRA that led to a clearer depiction of vessels. Therefore, image quality could be further improved by the development of new techniques.

However, some limitations of this study should be recognized. First, because of the high accuracy of CTA and the disadvantages of DSA, CTA was used as the reference in this study. Furthermore, DSA is not generally routinely used for screening cerebrovascular diseases, and it would be very difficult to obtain approval of the ethics committee to evaluate this issue in our patient population. However, DSA would be more accurate if used as the reference. Second, contrast-enhanced MRA was not performed as a comparison method because there is some concern regarding the combined adverse effects of MR imaging and CTA contrast media on renal function. In addition, we aimed to investigate the validation of zTE-MRA in noncontrast MRA sequences. Third, when scan time was compromised, the resolution of peripheral vessels by zTEMRA seemed to be lower than that achieved by TOF-MRA, though larger coverage was obtained, and cerebrovascular lesions were visualized much better on zTE-MRA. The modification of some parameters would improve the resolution of zTE-MRA in future studies. Fourth, the data obtained for objective sound-level measurements do not reflect the real situation inside the coil, though noise levels were statistically lower during the zTE-MRA scan. More advanced and accurate measurement techniques must be used in future studies.

\section{CONCLUSIONS}

In this study, we found that compared with TOF-MRA, zTEMRA achieved higher image quality and accuracy, provided a more comfortable experience for the patient, and produced results more consistent with those obtained using CTA. Due to its 
robust performance, zTE-MRA is demonstrated to be a promising non-contrast-enhanced alternative MRA technique that could be more routinely used in the clinic for patients with cerebrovascular diseases. Future studies should also provide supporting evidence and verification in larger clinical populations for zTE-MRA and should also include additional varieties of diseases.

\section{REFERENCES}

1. Hollnagel DI, Summers PE, Poulikakos D, et al. Comparative velocity investigations in cerebral arteries and aneurysms: 3D phasecontrast MR angiography, laser Doppler velocimetry and computational fluid dynamics. NMR Biomed 2009;22:795-808 CrossRef Medline

2. Lavina B. Brain vascular imaging techniques. Int J Mol Sci 2016;18 CrossRef Medline

3. Li Q, Lv F, Wei Y, et al. Automated subtraction CT angiography for visualization of the whole brain vasculature: a feasibility study. Acad Radiol 2013;20:1009-14 CrossRef Medline

4. Zhao DL, Wan Y, Wang GK, et al. Evaluation of image quality in carotid and cerebrovascular disease: a comparative study between subtraction and routine computed tomography angiography. Echocardiography 2016;33:1735-40 CrossRef Medline

5. Roth C. Cerebrovascular diagnostics: imaging [in German]. Radiologe 2012;52:1101-06 CrossRef Medline

6. Wu H, Block WF, Turski PA, et al. Noncontrast-enhanced threedimensional (3D) intracranial MR angiography using pseudocontinuous arterial spin labeling and accelerated 3D radial acquisition. Magn Reson Med 2013;69:708-15 CrossRef Medline

7. Irie R, Suzuki M, Yamamoto M, et al. Assessing blood flow in an intracranial stent: a feasibility study of MR angiography using a silent scan after stent-assisted coil embolization for anterior circulation aneurysms. AJNR Am J Neuroradiol 2015;36:967-70 CrossRef Medline

8. Takano N, Suzuki M, Irie R, et al. Usefulness of non-contrast-enhanced MR angiography using a silent scan for follow-up after Yconfiguration stent-assisted coil embolization for basilar tip aneurysms. AJNR Am J Neuroradiol 2017;38:577-81 CrossRef Medline

9. Takano N, Suzuki M, Irie R, et al. Non-contrast-enhanced silent scan MR angiography of intracranial anterior circulation aneurysms treated with a low-profile visualized intraluminal support device. AJNR Am J Neuroradiol 2017;38:1610-16 CrossRef Medline

10. Shang S, Ye J, Luo X, et al. Follow-up assessment of coiled intracranial aneurysms using zTE MRA as compared with TOF MRA: a preliminary image quality study. Eur Radiol 2017;27:4271-80 CrossRef Medline

11. Yang L, Huang X, Duan S. Clinical application and technique of 64-slice spiral CT subtraction angiography in head and neck. Vasa 2012;41:27-33 CrossRef Medline

12. Alibek S, Vogel M, Sun W, et al. Acoustic noise reduction in MRI using Silent Scan: an initial experience. Diagn Interv Radiol 2014;20: 360-63 CrossRef Medline

13. Cong F, Zhuo Y, Yu S, et al. Noncontrast-enhanced time-resolved 4D dynamic intracranial MR angiography at 7T: a feasibility study. J Magn Reson Imaging 2018;48:111-20 CrossRef Medline
14. Kim YK, Lin WC, Sung K, et al. Reducing artifacts during arterial phase of gadoxetate disodium-enhanced MR imaging: dilution method versus reduced injection rate. Radiology 2017;283:429-37 CrossRef Medline

15. Boujan T, Neuberger U, Pfaff J, et al. Value of contrast-enhanced MRA versus time-of-flight MRA in acute ischemic stroke MRI. AJNR Am J Neuroradiol 2018;39:1710-16 CrossRef Medline

16. HaiFeng L, YongSheng X, YangQin X, et al. Diagnostic value of 3D time-of-flight magnetic resonance angiography for detecting intracranial aneurysm: a meta-analysis. Neuroradiology 2017;59:1083-92 CrossRef Medline

17. Fujima N, Osanai T, Shimizu Y, et al. Utility of noncontrast-enhanced time-resolved four-dimensional MR angiography with a vessel-selective technique for intracranial arteriovenous malformations. J Magn Reson Imaging 2016;44:834-45 CrossRef Medline

18. Togao O, Hiwatashi A, Obara M, et al. Acceleration-selective arterial spin-labeling MR angiography used to visualize distal cerebral arteries and collateral vessels in Moyamoya disease. Radiology 2018; 286:611-21 CrossRef Medline

19. Igase K, Igase M, Matsubara I, et al. Mismatch between TOF MR angiography and $\mathrm{CT}$ angiography of the middle cerebral artery may be a critical sign in cerebrovascular dynamics. Yonsei Med J 2018;59: 80-84 CrossRef Medline

20. Mcnulty JP, Mcnulty S. Acoustic noise in magnetic resonance imaging: an ongoing issue. Radiography 2009;15:320-26 CrossRef

21. Mollasadeghi A, Mehrparvar AH, Atighechi S, et al. Sensorineural hearing loss after magnetic resonance imaging. Case Rep Radiol 2013;2013:510258 CrossRef Medline

22. Munn Z, Jordan Z. Interventions to reduce anxiety, distress and the need for sedation in adult patients undergoing magnetic resonance imaging: a systematic review. Int J Evid Based Healthc 2013;11: 265-74 CrossRef Medline

23. Weiger M, Brunner DO, Dietrich BE, et al. ZTE imaging in humans. Magn Reson Med 2013;70:328-32 CrossRef Medline

24. Holdsworth SJ, Macpherson SJ, Yeom KW, et al. Clinical evaluation of silent T1-weighted MRI and silent MR angiography of the brain. AJR Am J Roentgenol 2018;210:404-11 CrossRef Medline

25. Moon JI, Baek HJ, Ryu KH, et al. A novel non-contrast-enhanced MRA using silent scan for evaluation of brain arteriovenous malformation: a case report and review of literature. Medicine (Baltimore) 2017;96:e8616 CrossRef Medline

26. Choi CG, Lee DH, Lee JH, et al. Detection of intracranial atherosclerotic steno-occlusive disease with $3 \mathrm{D}$ time-of-flight magnetic resonance angiography with sensitivity encoding at 3T. AJNR Am J Neuroradiol 2007;28:439-46 Medline

27. Weiger $\mathrm{M}, \mathrm{Wu} \mathrm{M}$, Wurnig $\mathrm{MC}$, et al. Rapid and robust pulmonary proton ZTE imaging in the mouse. NMR Biomed 2014;27:1129-34 CrossRef Medline

28. Dai W, Garcia D, Bazelaire CD, et al. Continuous flow-driven inversion for arterial spin labeling using pulsed radio frequency and gradient fields. Magnet Reson Med 2008;60:1488-97 CrossRef Medline

29. Qu J, Wu B, Zhou Z. Silent magnetic resonance angiography with hybrid arterial spin labeling techniques. In: Proceedings of the Annual Meeting of the International Society for Magnetic Resonance in Medicine, Singapore. May 7-13, 2016 